\title{
Pervasive read-through transcription of $T$ - DNAs is frequent in tobacco BY-2 cells and can effectively induce silencing
}

\author{
Vojtěch Čermák and Lukáš Fischer *i
}

\begin{abstract}
Background: Plant transformation via Agrobacterium tumefaciens is characterized by integration of commonly low number of T-DNAs at random positions in the genome. When integrated into an active gene region, promoterless reporter genes placed near the T-DNA border sequence are frequently transcribed and even translated to reporter proteins, which is the principle of promoter- and gene-trap lines.

Results: Here we show that even internal promotorless regions of T-DNAs are often transcribed. Such spontaneous transcription was observed in the majority of independently transformed tobacco BY-2 lines (over 65\%) and it could effectively induce silencing if an inverted repeat was present within the T-DNA. We documented that the transcription often occurred in both directions. It was not directly connected with any regulatory elements present within the T-DNAs and at least some of the transcripts were initiated outside of the T-DNA. The likeliness of this read-through transcription seemed to increase in lines with higher T-DNA copy number. Splicing and presence of a polyA tail in the transcripts indicated involvement of Pol II, but surprisingly, the transcription was able to run across two transcription terminators present within the T-DNA. Such pervasive transcription was observed with three different T-DNAs in BY-2 cells and with lower frequency was also detected in Arabidopsis thaliana.

Conclusions: Our results demonstrate unexpected pervasive read-through transcription of T-DNAs. We hypothesize that it was connected with a specific chromatin state of newly integrated DNA, possibly affected by the adjacent genomic region. Although this phenomenon can be easily overlooked, it can have significant consequences when working with highly sensitive systems like RNAi induction using an inverted repeat construct, so it should be generally considered when interpreting results obtained with the transgenic technology.
\end{abstract}

Keywords: GFP, Inverted repeat, Promoterless, RNAi, Read-through transcription, T-DNA, Tobacco BY-2 cell line

\section{Background}

Agrobacterium tumefaciens mediated transformation is a common method used to obtain transgenic plants. Agrobacterium transfers its T-DNA into a plant cell, where it can be integrated inside plant genome predominantly through double-strand break repair pathway (reviewed in [1]). Generally T-DNAs are stably introduced only in a small proportion of cells cocultivated with Agrobacterium. The T-DNAs are integrated at random positions in their genome [2, 3]. Subsequent regeneration of transgenic lines requires incorporation of a selection step to filter out untransformed cells/

\footnotetext{
* Correspondence: lukas.fischer@natur.cuni.cz

Department of Experimental Plant Biology, Charles University, Faculty of Science, Viničná 5, 12844 Prague 2, Czech Republic
}

plants. This selection is usually achieved by incorporating antibiotic or herbicide resistance gene into the T-DNA. The requirement for the selection gene to be actively expressed then imposes bias on the selected transformants. T-DNAs of such transformants are preferentially present in regions with active transcription, especially near promoters and in regions with low nucleosome density [3, 4]. This probably leads to the unusually high success rate of various promoter- and gene-trap lines [5-7]. Although the transformants generated by Agrobacterium have lower number of insertions compared to other transformation methods, there are still many transformants with multiple insertions. Commonly the number of insertions per line varies between 1.4 and $4.9[4,8]$. In case of multiple insertions, it is quite common for T-DNAs to integrate in a form of direct or inverted

(c) The Author(s). 2018 Open Access This article is distributed under the terms of the Creative Commons Attribution 4.0 International License (http://creativecommons.org/licenses/by/4.0/), which permits unrestricted use, distribution, and 
repeats in one position in the genome. Due to the way how $T-D N A s$ are integrated, the most common form is the head-to-head (RB-to-RB) inverted repeat arrangement [9, 10]. Convergent read-through transcription of T-DNAs integrated as inverted repeats can induce silencing of homologous sequences via RNA interference (RNAi) [11, 12].

RNAi is an important mechanism in the regulation of gene expression in eukaryotic cells. In functional genomics, it is often used as a tool to modify expression of studied genes. The key players in RNAi are small RNAs (sRNAs), which can be formed by multiple pathways in plants, making the plant RNAi a very complex process (reviewed in [13]). Generally, a double stranded RNA (dsRNA) is needed to induce sRNA production in plants. There are many different ways to achieve dsRNA formation in a cell, the most efficient one is intermolecular pairing of transcripts coming from an inverted repeat [14].

Triggering RNAi by introduced "silencer constructs" can be used to knock out genes of interest or to study the mechanisms of RNAi itself. The large majority of RNAi studies were based on the model plant Arabidopsis thaliana that offers high quality genomic data and plenty of mutant lines, which are easily accessible to the research community. Few years ago, we started to test an alternative model, tobacco BY-2 cell line that has been successfully used in numerous studies focused on cellular processes [15]. The BY-2 cell culture is composed of relatively homogeneous mitotically proliferating cells $[15,16]$. The absence of the gametophytic phase also prevents some types of epigenetic changes connected with this developmental stage [17-20]. The BY-2 cell line also allows simple analyses at the level of individual cells and assessment of a large number of independent transgenic lines (in the callus form) that can be easily generated, managed and analyzed [16]. Since the behavior of individual transgenic lines of any model is affected by the T-DNA copy number and the chromosomal environment of the insertion [21-23], analysis of a high number of independent transformed lines, which provides a more generalized picture, is recommended. Other advantages include easy and reliable analyses of fluorescence levels in these cells as well as simple ways to treat these cells with various chemicals. In situations, where the study is focused on analyses of general molecular and cellular mechanisms, the absence of the whole plant context may not have substantial impact on the appropriate generalization of the results.

The observation of pervasive read-through transcription of T-DNAs that we describe in this study, was discovered during our RNAi project focused on comparison of silencing potential of different silencing inducers. We supertransformed a BY-2 line stably expressing the GFP gene [24] with various silencers that were not controlled by a constitutive promoter as usual, but they were based on the XVE inducible system $[25,26]$, providing the possibility of highly reliable induction of RNAi by $\beta$-estradiol. The GFP reporter gene was used to allow simple visualization of silencing.

We generated hundreds of independent transgenic BY-2 lines (calli) with a goal to assess population responses to the induced expression of each silencing inducer (these data are not presented in this study). Surprisingly, we observed significant differences between the silencers already prior to their activation with $\beta$-estradiol with high frequency of silencing occurring in the calli carrying the inverted repeat construct. We found that this silencing correlated with spontaneous transcription of the silencer and here we show detailed analysis of this phenomenon.

\section{Methods}

\section{Plasmid construction}

All silencer T-DNAs (Fig. 1) were prepared similarly. First the full length GFP sequence was PCR amplified from psmRS-GFP plasmid [27] using primers with appropriate adapters (Additional file 1). The sequence was then inserted into the $p$ Drive vector (part of QIAGEN PCR Cloning Kit) and subsequently transferred into the destination binary vector - either $p E R 8$ [26] or pGreen [28] using the appropriate restriction endonucleases. The $A S$-GFP was cloned between $X h o \mathrm{I}$ and $B c u \mathrm{I}$ in $p E R 8$, the $U T$-GFP was cloned between $X h o \mathrm{I}$ and $P v u \mathrm{II}$ in $p E R 8$, the GFP was cloned between $X h o \mathrm{I}$ and $B c u \mathrm{I}$ in $p E R 8$ (as SalI and NheI fragment derived from $p D r i v e ~ I R-G F P$ ). The $I R-G F P$ construct was first assembled in pDrive: the intron from Solanum tuberosum PsbO gene (cDNA GeneBank no. X17578.1) including approximately $20 \mathrm{bp}$ from exons on both sides was AT-cloned into pDrive (in SacI-KpnI orientation). The intron sequence placed inside the inverted repeats was demonstrated to enhance the efficiency of silencing likely via facilitating dsRNA formation [29]. IR1-GFP was inserted first between XhoI and SalI, then IR2-GFP was inserted between PstI and BamHI; the whole IR-GFP construct was then cloned between XhoI and $B c u \mathrm{I}$ in $p E R 8$. The IR-GFP- $\triangle P 1$ control was created by cloning the IR-GFP construct between $\mathrm{SalI}$ and $B c u \mathrm{I}$ in $p E R 8$ (SalI cleaves at the beginning of the inducible promoter). The IR-GFP- $\triangle P 2$ control was created by cloning the IR-GFP construct in pGreen 0129 between SacI and $K p n I$. All cloning experiments were completed using enzymes from Fermentas (Thermo Fisher Scientific) and Escherichia coli strain JM109. The resulting T-DNAs were confirmed by restriction and sequencing (Fig. 1).

\section{Plant material}

Tobacco cell line BY-2 (Nicotiana tabacum L. cv. Bright Yellow 2) [15] was obtained from Prof. Zdeněk Opatrný, who had cultivated the line for more than 20 years. BY-2 calli were cultivated on agar plates $(0.8 \mathrm{w} / v$ agar; $6 \mathrm{~cm}$ 


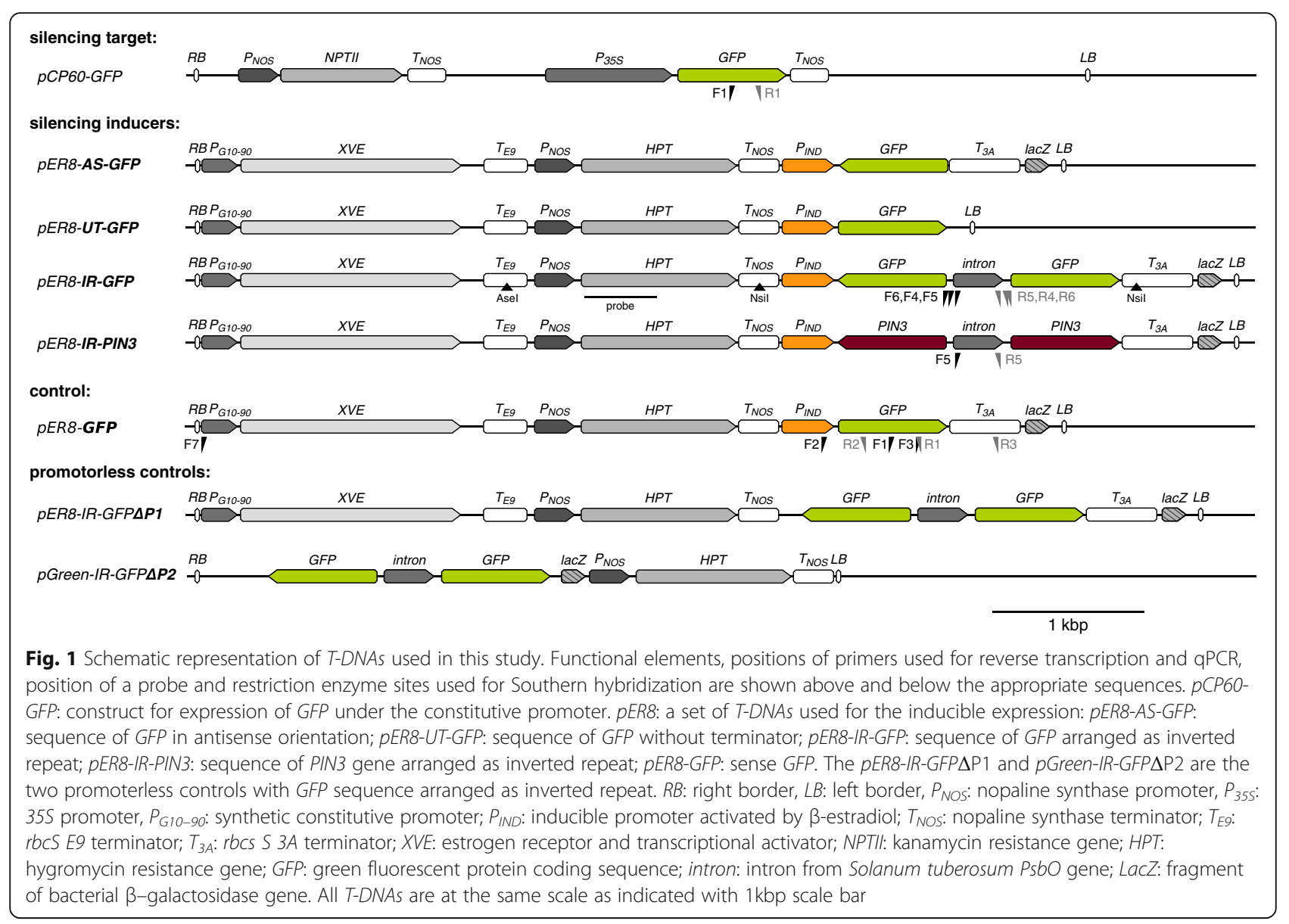

diameter plates) with modified MS medium [30]. The calli were subcultured monthly. Suspension cell cultures were subcultured every seventh day $(1 \mathrm{ml}$ of cells into $30 \mathrm{ml}$ of liquid media). The cultures were kept in darkness at $26{ }^{\circ} \mathrm{C}$; suspensions were placed on the orbital shaker IKA KS501 at 110 rpm (IKA Labortechnik, Staufen, Germany; orbital diameter $30 \mathrm{~mm}$ ).

Transformations of BY-2 suspension cells were carried out as described previously [24] using Agrobacterium tumefaciens strain $\mathrm{C} 58 \mathrm{C} 1$ carrying a helper plasmid $p G V 2260$ [31] and appropriate binary vector (see above). After cocultivation with agrobacterium, the cells were plated on solidified medium containing $25 \mu \mathrm{g} / \mathrm{ml}$ hygromycin and $100 \mu \mathrm{g} / \mathrm{ml}$ cefotaxim and cultured for 3 weeks. Using this procedure, individual transformed cells form isolated macroscopic cell clusters (commonly called calli) that can be mostly regarded as genetically homogeneous clones [24].

The promoter from the $p E R 8$ plasmid was induced by cultivating the calli on media with addition of $2 \mu \mathrm{M}$ $\beta$-estradiol (from Sigma-Aldrich Cat. No. E2758). The $\beta$-estradiol was stored as $20 \mathrm{mM}$ solution in DMSO, therefore, a corresponding amount of DMSO was added to the cultivation medium of the controls.
Arabidopsis thaliana (Col-0) plants were grown in Jiffy soil pellets under long-day conditions with illumination of $100 \mu \mathrm{m} \mathrm{m}^{-2} \mathrm{~s}^{-1}$ photosynthetically active radiation (OSRAM L 58 W/930).

\section{Measurement and analysis of fluorescence level}

The BY-2 calli grown after transformation (see above) were transferred to new plates with $100 \mu \mathrm{g} / \mathrm{ml}$ cefotaxim (20 calli per plate) and cultivated for approximately 8 days. 200 calli (each representing different transgenic event) were used for each of the variants. Each plate was photo-documented separately using G:BOX (SynGene, Cambridge, UK) with blue excitation light (LED diodes with maximum at $\lambda=465 \mathrm{~nm}$ ) and green emission filter (FILT525/GX; 510-540 nm). The images were processed using software NIS-Elements 3.10 (Laboratory Imaging, Prague, CZ). The average light intensity was measured for all the pixels from each callus. These data were statistically analyzed using $\mathrm{R}$ 3.1.2 and Pearson's chi-square test. The threshold for fluorescent and non-florescent calli was set as the highest fluorescence intensity measured for wild-type BY-2 callus (these were used as controls cultivated alongside the transgenic calli). 


\section{Transcription analysis}

RNA was isolated from $100 \mathrm{mg}$ of 10 to 18 days old calli (in the same experiment the calli were always in the same age) or from $50 \mathrm{mg}$ of siliques of 40 days old Arabidopsis thaliana plants (younger siliques were selected - less than 10 days old, to ensure high proportion of dividing cells in the tissue) using NucleoSpin ${ }^{\circ}$ RNA Plant kit (MACHEREY-NAGEL, Düren, DE). The procedure was performed according to manufacturer's instructions including the on-column DNA digestion. The RNA was measured on NanoDrop 2000 (Thermo Fisher Scientific) to assess the concentration of the samples and to exclude contamination of the RNA by impurities. The integrity of the RNA was checked by gel electrophoresis using the "bleach gel" method [32]. For cDNA preparation, $1 \mu \mathrm{g}$ of the total RNA was again treated with DNase I and half of the reaction mixture was then used as template for RevertAid Reverse Transcriptase (Fermentas, Thermo Fisher Scientific). The other half was exposed to the same treatment, but without adding the Reverse Transcriptase - this served as a control to check for DNA contamination. The final cDNA was diluted into the volume of $50 \mu$ l. Either oligo(dT) or specific primers were used for the cDNA synthesis. Some of the specific primers were designed to allow for distinction between sense and antisense transcripts and spliced and unspliced molecules (Additional file 1).

The quantification itself was done by qPCR, using LightCycler 480 (Roche) and $\mathrm{iQ}^{\mathrm{m}} \mathrm{SYBR}^{\circ}$ Green Supermix (BioRad, Hercules, CA, USA). All the experiments were done while keeping the general qPCR guidelines in mind [33]. Reactions were completed in $10 \mu \mathrm{l}$ volume, using $1 \mu \mathrm{l}$ of cDNA as a template; all reactions were done in triplicate. The specificity of the PCR was verified by melting curve analysis (using the LightCycler 480 software) and also by checking randomly selected samples using gel electrophoresis. For each set of primers, there was appropriate negative control (WT and/or $\mathrm{dH}_{2} \mathrm{O}$ ). The PCR efficiency for each amplicon and the $\mathrm{Cq}$ values for each sample were calculated using the software LinRegPCR 2015.3 [34]. The values for triplicates were averaged after correction for PCR efficiency. Samples in the triplicate with no amplification or only unspecific products were counted as zeroes; the samples with the majority of unspecific product was treated as one order of magnitude smaller (unspecific products appeared only for samples with high Cq values, over 30). Calculated concentrations were normalized to the expression of $N t E F 1 \alpha$, so all the presented values show the relative level of given transcript to the level of NtEF1 $\alpha$. Results were then statistically compared using $\mathrm{R}$ 3.1.2 and Welch's t-test. Positions of primers used for qPCR are indicated in Fig. 1 and their sequences listed in Additional file 1. Some primer sequences were taken over from previous studies [35, 36].
The BY-2 calli for transcriptional analysis were selected randomly from groups of silenced and non-silenced calli based on the presence or absence of GFP fluorescence. In case of transformants carrying sense GFP (GFP sequence in $p E R 8$ XVE inducible system), the BY-2 calli and Arabidopsis lines were randomly selected from those that were able to induce GFP expression when grown on the induction medium supplemented with $\beta$-estradiol.

\section{Southern blot analysis}

The Southern blot hybridization was done as described previously [37] with the following modifications: The DNA was isolated from $150 \mathrm{mg}$ (FW) of BY-2 calli. $20 \mu \mathrm{g}$ of genomic DNA per sample was separately digested by enzymes NsiI and AseI (New England Biolabs). The probe was prepared as a fragment of HPT gene using PCR with primers HPT_probe_F and $H P T \_$probe_R (Additional file 1).

The Southern blot was interpreted as follows: tandem $T-D N A$ inserted as direct repeat should give $6.8 \mathrm{kbp}$ fragment with both NsiI and AseI, plus one fragment of unknown size for NsiI and AseI; head-to-head inverted repeat should give $7.3 \mathrm{kbp}$ fragment when digested with $N s i$ and two fragments of unknown size when digested with AseI; tail-to-tail inverted repeat should give $9.6 \mathrm{kbp}$ fragment when digested with AseI and two fragments of unknown size when digested with NsiI.

\section{Results}

\section{Fluorescence in calli transformed with various GFP silencer constructs}

To study various aspects of RNAi, we prepared three different silencer T-DNAs based on the XVE inducible system [26]. Specifically, the silencing should have been achieved through production of i) antisense RNA (AS-GFP), ii) non-polyadenylated sense RNA (GFP without any terminator; $U T-G F P$ ) and iii) hairpin RNA (inverted repeat with an intron separating the antisense and sense GFP fragment; $I R-G F P)$. These T-DNAs were expected to trigger posttranscriptional silencing of the reporter GFP gene only under induction with $\beta$-estradiol. As a control, we also prepared a construct with inducible GFP in sense orientation and ended with terminator (GFP; Fig. 1).

A selected BY-2 cell line that has been stably expressing GFP (driven by $35 S$ promoter) for more than 8 years [24] was separately supertransformed with each T-DNA. We then analyzed GFP fluorescence in individual calli grown after the transformation - each representing independent transformation event (see Methods for details). On the control medium, where the calli were not exposed to $\beta$-estradiol, we expected similar fluorescence in all populations (hundreds of calli) carrying various silencers. However, we observed that IR-GFP population had strikingly 
lower frequency of calli with detectable GFP fluorescence compared to the other silencer and control T-DNAs (Fig. 2). All the differences between frequencies of GFP-positive calli in the IR-GFP population and those with other constructs were statistically significant $\left(p<10^{-40}\right)$. It should also be noted that some of the smaller differences between the variants were found significant as well: the UT-GFP variant compared to EV and GFP $\left(p<10^{-6}\right)$.

Since the calli grew on the medium without $\beta$-estradiol, the observed unexpectedly high proportion (over 65\%) of spontaneously silenced IR-GFP calli prompted us to study this phenomenon further. Although XVE inducible system is considered to be reliable with very low leakiness of the inducible promoter [26], we had to exclude this possibility. We prepared two additional controls; i) we removed the inducible promoter from the $p E R 8$ vector (IR-GFP $\triangle P 1)$ and ii) we cloned the promoterless $I R-G F P$ into the empty $p$ Green vector (IR-GFP $\triangle P 2)$. After transformation to BY-2 cells, the number of spontaneously silenced independent calli was virtually the same as with the original IR-GFP T-DNA (Fig. 2, columns 5 and 6; the differences between the IR variants were not statistically significant), indicating that the silencing was independent of the presence of the inducible promoter and the T-DNA context.

To exclude the possibility that the transcription was initiated from elements that might be common for both T-DNAs, we compared their sequences. We found two homologous regions: i) the HPT expression cassette, which, however, differed between the two $T$-DNAs in its orientation relative to the IR-GFP sequence (Fig. 1) and ii) a short 156-nt fragment of bacterial $\beta$-galactosidase gene $(\operatorname{LacZ})$ that was downstream of the IR-GFP in both T-DNAs. No promoter regulatory elements were predicted within this sequence by TSSP software (http://linux1.softberry.com).

\section{Transcription and splicing of IR-GFP}

Transcription analysis was done in five non-silenced and five silenced calli that were randomly selected from populations with and without detectable GFP fluorescence. The results showed that the levels of GFP transcripts roughly matched the GFP fluorescence intensities, with the lowest transcription being detected in the silenced calli (Fig. 3a and b). To see whether the silencing correlated with transcription of the IR-GFP, we generated cDNAs using primers specific for the GFP hairpin transcribed in both the sense and antisense orientation (the "sense" and "antisense" transcripts were relative to the intron separating the GFP sequences in the IR-GFP). Transcript levels were analyzed using qPCR (Fig. 3b-h) with primers designed to allow separate quantification of i) spliced transcripts, ii) unspliced transcripts and iii) intron-containing molecules (i.e. nascent transcripts and spliced introns; Fig. 1). The $I R$-GFP transcripts were detected in all the spontaneously silenced calli at levels even higher than in the callus, where the IR-GFP transcription was induced with $\beta$-estradiol. In contrast, almost undetectable levels (three to four orders of magnitude lower) were found in all non-silenced calli with detectable GFP fluorescence (Fig. 3a and c, for the comparison of averaged relative transcript levels and their statistical comparison see Additional file 2). Surprisingly, in three of the five silenced calli, the $I R-G F P$ was clearly transcribed also in the "antisense" direction (from the terminator towards the promoter), although at lower levels than it was

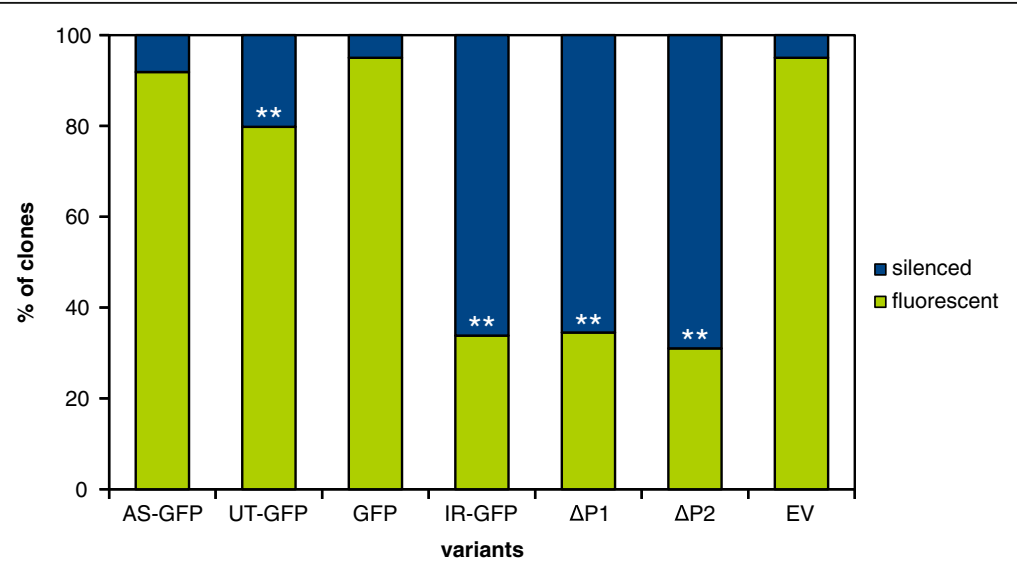

Fig. 2 Frequency of spontaneous silencing of GFP in supertransformed BY-2 calli. Silencer and control T-DNAs (as described in Fig. 1) were supertransformed to BY-2 cell line stably expressing GFP. The fluorescence was assessed in independently transformed calli grown on noninductive medium six weeks after transformation. The bars represent the percentage of fluorescent non-silenced calli (the lower part of the bar) and silenced calli (the upper part of the bar). The variants AS-GFP and UT-GFP represent averages of three biological replicas and the variants GFP and IR-GFP represent averages of four biological replicas (independent transformations). Each replica had 200 calli (with exception of EV with 160 calli). Variants that significantly differed from the EV control are marked with ${ }^{* *}\left(p<10^{-6}\right) . \Delta P 1$ : pER8-IR-GFPAP1; $\triangle P 2$ : pGreen-IR-GFPAP2; EV: empty vector $p E R 8$ 

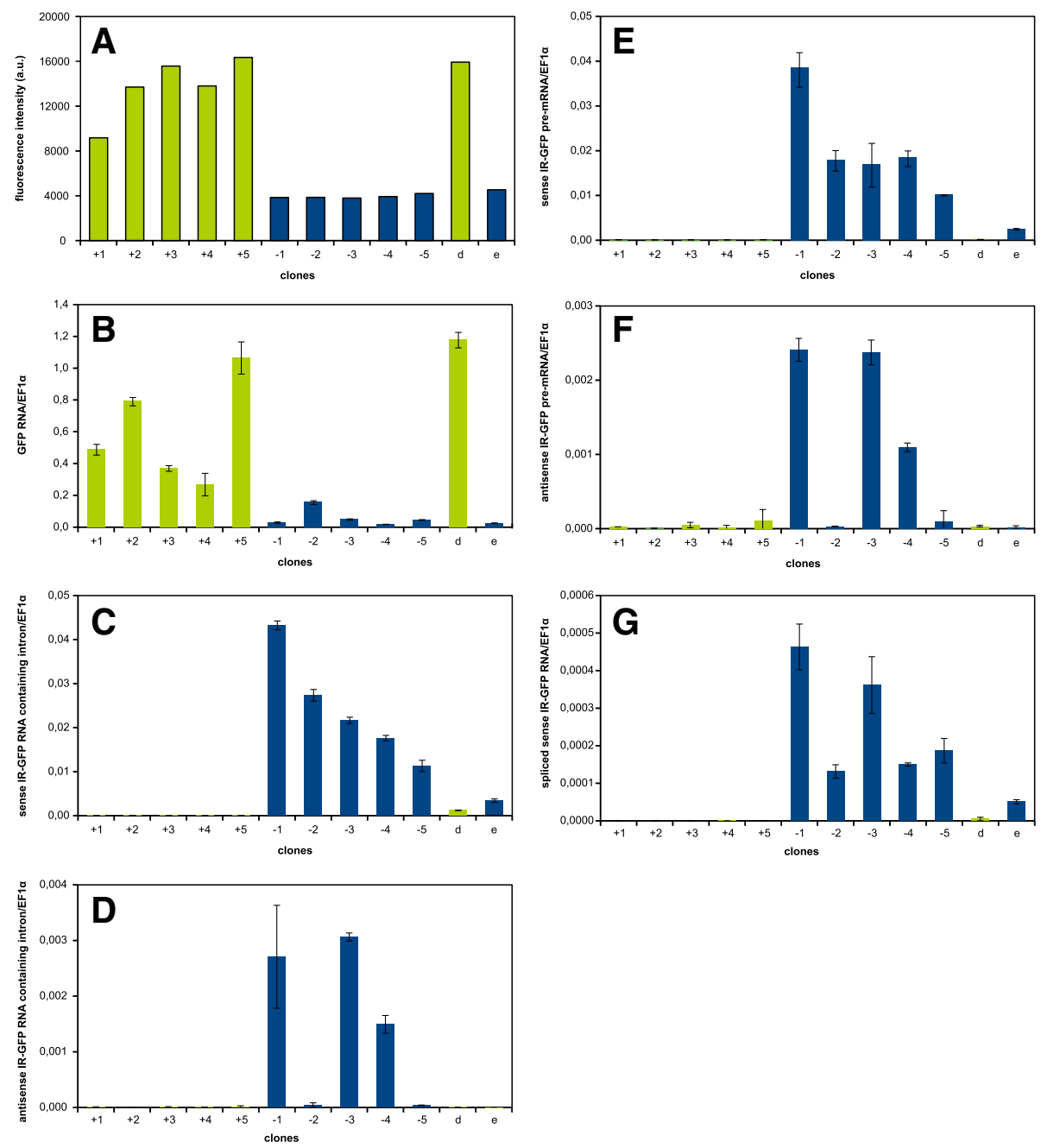

Fig. 3 Analysis of selected BY-2 calli supertransformed with pER8-IR-GFP. RT-qPCR analysis of IR-GFP transcript levels in five randomly selected spontaneously silenced calli (marked from -1 to -5 ) and five non-silenced calli (marked from +1 to +5 ) and one additional non-silenced callus grown on the induction medium with $\beta$-estradiol (marked as " $e$ ") and on the control medium with DMSO (marked as "d"). Each callus represented independent transformation event. a Fluorescence of analyzed calli (in arbitrary units), signals below 4000 represent the background; b the level of the sense GFP transcript (RT: oligo dT primer, QPCR: F1 and R1 primers); $\mathbf{c}$ the level of transcripts with the sense intron of IR-GFP (representing both the unspliced transcript and the spliced intron; RT: R5 primer, QPCR: F5 and R5 primers); $\mathbf{d}$ the level of transcripts with the antisense intron of IR-GFP (RT: F5 primer, QPCR: F5 and R5 primers); e the level of the sense IR-GFP unspliced transcript (RT: R5 primer, qPCR: F4 and R5 primers); $\mathbf{f}$ the level of the antisense IR-GFP unspliced transcript (RT: F5 primer, qPCR: F5 and R4 primers); $\mathbf{g}$ the level of the sense spliced transcript (RT: R6 primer, qPCR: F6 and R6 primers)

transcribed in the "sense" orientation. There was no impact of $\beta$-estradiol treatment on the hairpin transcription in the antisense orientation in the control line (Fig. 3d).

The results obtained from detecting the intron sequence were similar to those obtained from detecting the unspliced transcript (PCR product spanning the exon-intron boundary), this was also true for the antisense direction (Fig. 3e and f). Such results could indicate either fast degradation of the spliced intron or that the intron was not spliced at all. To see if the splicing actually took place, we ran $\mathrm{qPCR}$ over the supposed exon-exon boundary. Using the "sense" cDNA, we were indeed able to amplify the specific product corresponding to the spliced hairpin RNA in the silenced calli at similar levels in both the spontaneously silenced calli and in the estradiol-induced control. The ratio of spliced/unspliced transcripts was between 0.7 and $2.1 \%$ for the spontaneously transcribed lines and $2.1 \%$ for the estradiol-induced control. It suggested that at least some of the IR-GFP transcripts were spliced correctly in the spontaneously silenced calli (Fig. 3g; the specificity of the qPCR was verified, see Methods). 


\section{Spontaneous transcription of IR-PIN3 and sense GFP}

The spontaneous transcription and subsequent silencing could be specific for our IR-GFP construct or it could represent more general phenomenon. Therefore, we tested the occurrence of spontaneous transcription of another inverted repeat construct, IR-PIN3 prepared from a fragment of tobacco PIN3 gene placed in the $p E R 8$ plasmid (NtPIN3bT; kindly provided by Jan Petrášek) that we used to transform wild-type BY-2 cells. We analyzed the expression of the native PIN3 gene and the PIN3 hairpin in 11 randomly selected independent calli using RT-qPCR. We observed expression of the PIN3 hairpin in the majority of analyzed calli. The relative transcription levels seemed to be somewhat lower (from 2 to 20 times lower) compared to the GFP hairpin in the IR-GFP silenced calli (Fig. 4a-c, for the comparison of averaged relative transcript levels see Additional file 2). However, the comparison was based on the amplification of the intron sequence, so the real transcription levels of the two hairpins could differ due to various efficacy of their splicing. The IR-PIN3 transcription did not cause strong silencing of PIN3 in contrast to the situation with
IR-GFP and GFP. Only a weak decrease in PIN3 expression could be observed in some calli with the highest level of the PIN3 hairpin (see Fig. 4a and b). However, the averaged IR-PIN3 transcript levels did not significantly differ between calli with higher and lower PIN3 transcription (Additional file 2).

To assess if the observed high frequency of spontaneous transcription was specifically connected with inverted repeat arrangement of introduced transgenes or if it was more general phenomenon in our experimental system, we analyzed T-DNA transcription in lines carrying sense GFP in $p E R 8$ XVE inducible system (Fig. 1). After transformation into wild-type BY-2 cell line, there were no calli with detectable GFP fluorescence that would indicate spontaneous transcription connected with subsequent translation into functional GFP protein without $\beta$-estradiol treatment. However, RT-qPCR analysis showed that, at the transcriptional level, the sense GFP construct behaved similarly to the inverted repeats. The transcription was detected in all five calli and in both sense and antisense directions. The relative transcript levels were similar to those described for the
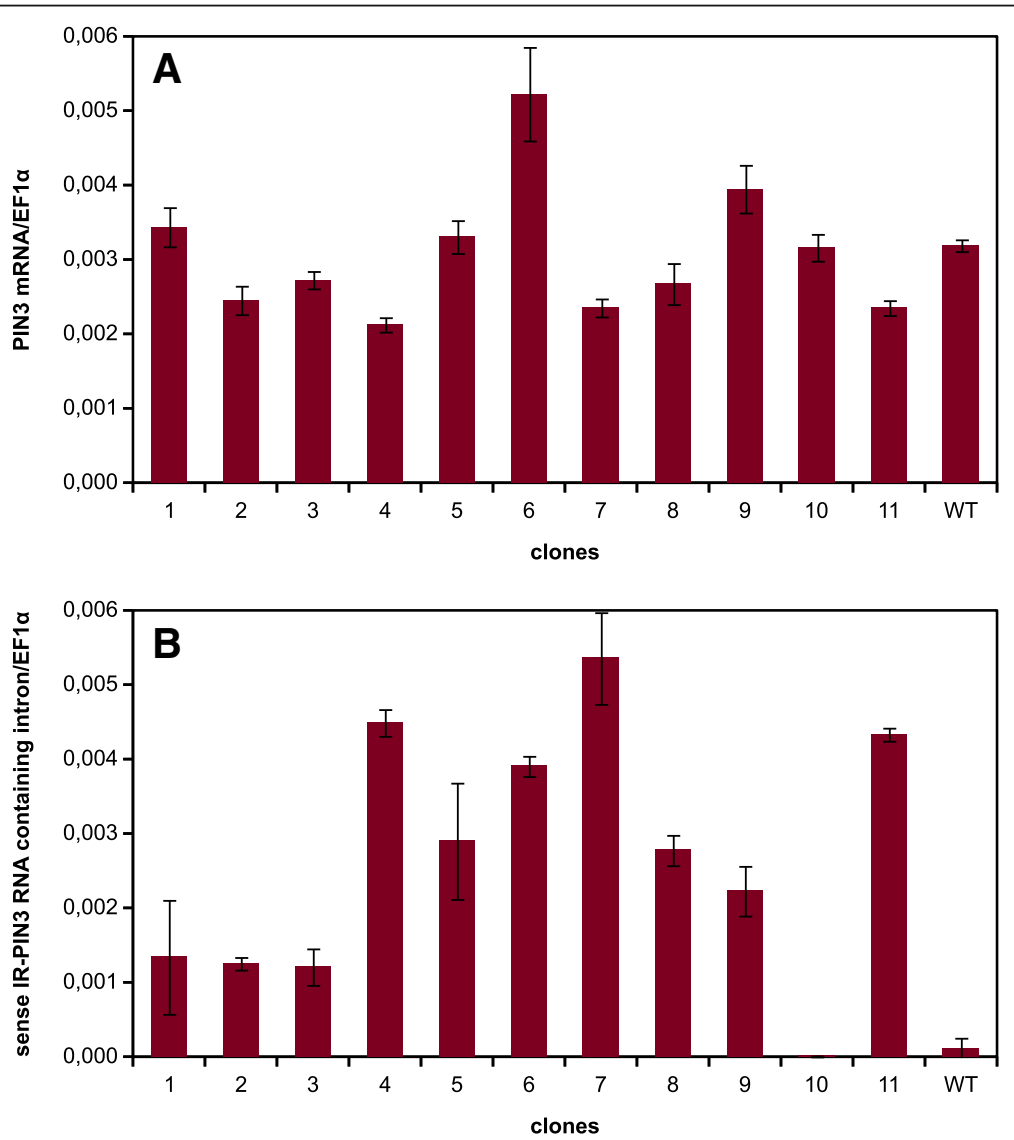

Fig. 4 Transcription analysis of selected BY-2 calli transformed with pER8-IR-PIN3. RT-qPCR analysis of transcript levels in eleven randomly selected calli and an untransformed BY-2 callus as a control. a The level of the PIN3 mRNA (RT: oligo dT primer, qPCR: PIN3_F and PIN3_R primers); $\mathbf{b}$ the level of the sense intron of the IR-PIN3 (representing both the unspliced transcript and the spliced intron; RT: R5 primer, qPCR: F5 and R5 primers) 
hairpins (Fig. 3c-f, Fig. 5a and c, Additional file 2). This showed that the transcription was not connected with the inverted-repeat character of the sequence present in the T-DNA.

To better understand the nature of the spontaneous transcription, we tried to identify the transcription start site by 5'RACE, but the attempts failed despite intensive optimization. Therefore, we investigated whether the transcript originated within or outside the transcription unit in $p E R 8 T-D N A$. For this purpose, we used a primer that matched to the region $50 \mathrm{nt}$ upstream of the transcription start site (TSS) for the sense transcript and a primer matching to the $3 \mathrm{~A}$ terminator region $50 \mathrm{nt}$ downstream of the last predicted poly(A) signal for the antisense transcript (as predicted by PASPA software: http://bmi.xmu.edu.cn/ paspa/index.html; [38]). We detected transcripts from both regions and their levels in individual calli correlated with previously detected transcripts of the GFP gene. This suggested that the spontaneous transcription originated (at least partially) outside of the transcription unit in $p E R 8$ $T-D N A$. The nearest ATG is 99 bp upstream from the proper $A T G$, so transcripts originating from this 99-bp long region could be theoretically translated into the proper GFP protein, but we have never detected GFP fluorescence in such lines, which indicated TSS being more upstream. Thus, we tried to roughly localize the position of TSS within the T-DNA. We designed a set of forward primers along the T-DNA for amplification from $c D N A$ prepared with a reverse primer specific to the GFP sequence. Surprisingly, we obtained products even with the most upstream primer located near the border sequence and preceding any promoter present in the T-DNA (Fig. 6). This PCR product
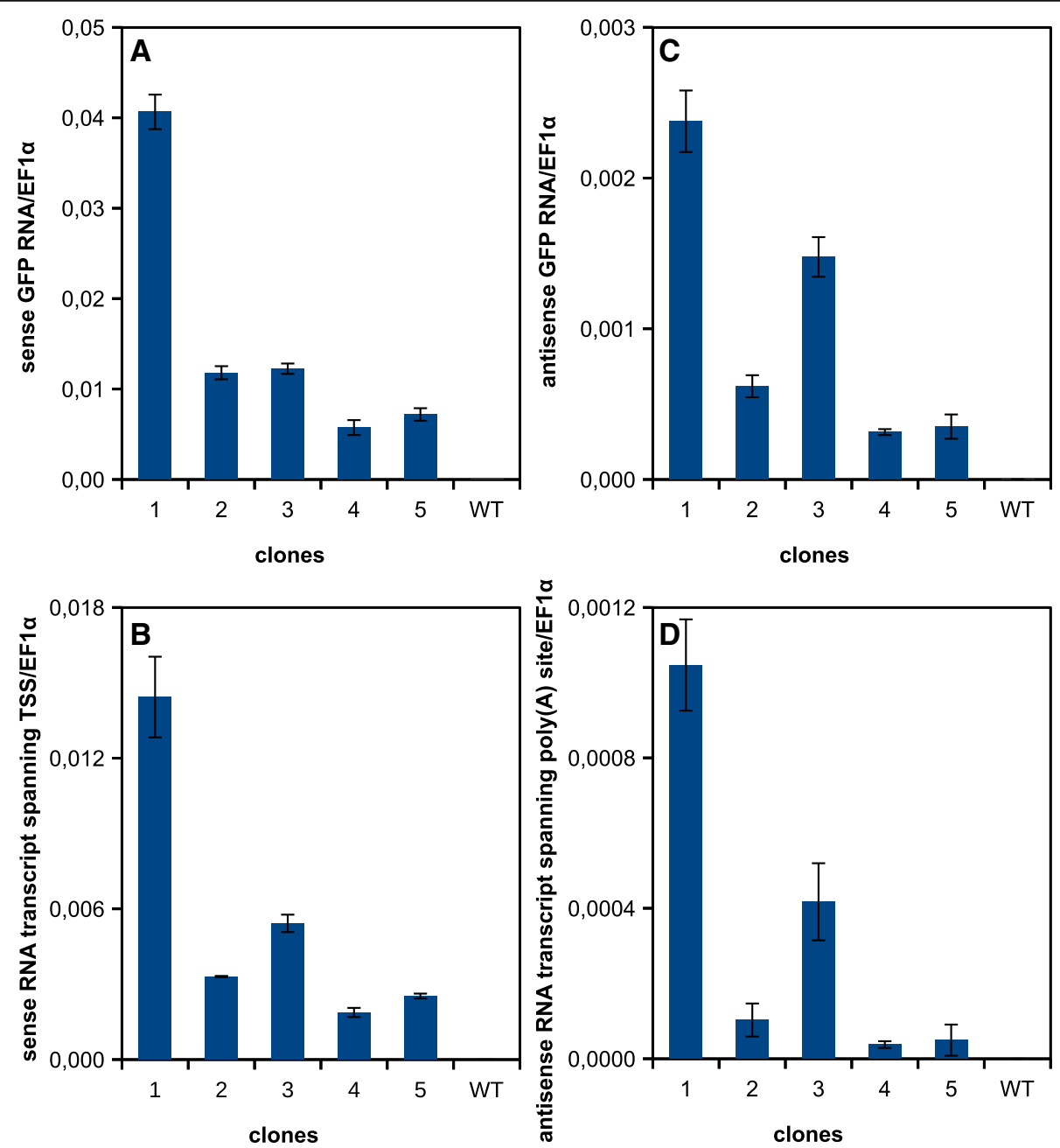

Fig. 5 Transcription of GFP gene in selected BY-2 calli transformed with pER8-GFP. RT-qPCR analysis of transcript levels in five selected independent calli grown on the medium without $\beta$-estradiol. a The level of the sense GFP transcript (RT: R1 primer, qPCR: F1 and R1 primers); $\mathbf{b}$ the level of the sense transcript containing the region $50 \mathrm{nt}$ upstream of transcription start site (TSS) of the inducible promoter (RT: R1 primer, qPCR: F2 and R2 primers); $\mathbf{c}$ the level of the antisense GFP transcript (RT: F1 primer, QPCR: F1 and R1 primers); $\mathbf{d}$ the level of the antisense transcript containing the region 50 nt downstream of the last poly(A) signal of T3A terminator (RT: F1 primer, QPCR: F3 and R3 primers) 


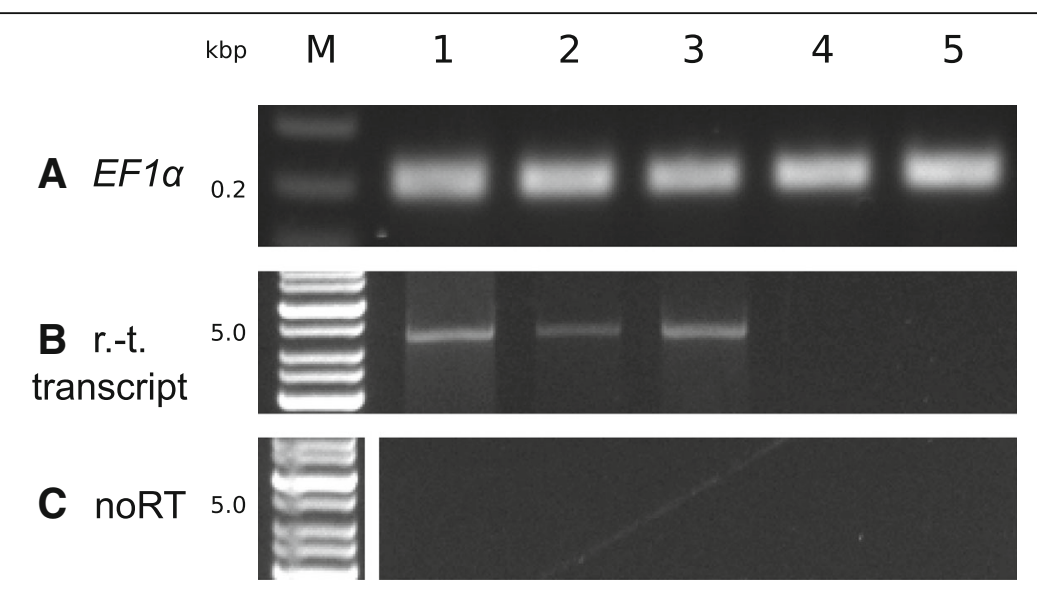

Fig. 6 Read-throught transcripts of the pER8 T-DNA in selected BY-2 calli transformed with pER8-GFP. Semiquantitative RT-PCR analysis of transcript levels in five independent calli untreated with $\beta$-estradiol (the same calli as in Fig. 5). cDNA was prepared using R1 primer. a The level of the EF1a transcript (internal standard); $\mathbf{b}$ the level of read-through (r.-t.) pER8 T-DNA transcript (F7 and R2 primers); c amplification of RNA samples that were not treated with reverse transcriptase to ensure that there was no DNA contamination

was more than $4 \mathrm{kbp}$ long and included two upstream transcription units for the $X V E$ receptor and the $H P T$ gene. Thus the transcription was able to overcome two transcription terminators (Fig. 1).

\section{Determination of T-DNA copy number and arrangement}

To better understand potential reasons of this transcription, we estimated copy number and arrangement of $T$-DNAs by Southern hybridization in three silenced and three non-silenced lines (selected according to the data in Fig. 3). The results indicated that the read-through transcription and spontaneous silencing might be connected with higher $T$-DNA copy number (Additional file 3); 1 to 3 copies were detected in non-silenced lines and 3 to approximately 8 copies in silenced lines. Further analyzes of the size and number of hybridizing bands in individual lines suggested that T-DNA arrangements allowing read-through transcription from one T-DNA to another, i.e. direct $T-D N A$ repeats and inverted tail-to-tail repeats, were not present in lines -1 and +2 . In the other four lines the situation was not clear due to multiple insertions $(-3,-4,+3)$ or the presence of truncated copies $(-4,+$ $1)$, but theoretically direct repeat could be present in lines -3 and -4 and tail-to-tail repeat in line -4 and +3 .

\section{Spontaneous transcription in Arabidopsis thaliana}

To assess wider significance of our observation we analyzed the transcription in a different model organism Arabidopsis thaliana plants carrying the same T-DNA with sense GFP in $p E R 8$ XVE inducible system. We randomly selected five transformants as before. RNA was isolated from immature siliques of plants grown in soil without any exposure to $\beta$-estradiol. As in the experiment with BY-2 cell line, we analyzed the GFP transcription in both the sense and antisense directions and also the transcripts from the regions spanning the canonical transcription start site and the poly(A) signal. We were able to detect transcripts in the sense direction from both the GFP sequence and from the region spanning the TSS and in the antisense direction from the GFP region (Fig. 7a-c). However transcription was detected in only one transformed line at the level comparable to the BY-2 callus with the lowest transcript level. The transcripts in the antisense direction over the poly(A) signal were almost undetectable (Fig. 7d). Transcription in leaves was somewhat lower (Additional file 4) than in immature siliques, which have higher proportion of actively dividing cells (similarly to the BY-2 cell line).

\section{Discussion}

\section{GFP silencing was connected with spontaneous} transcription of IR-GFP T-DNA

This study was prompted by observation of massive GFP silencing occurring after the introduction of IR-GFP construct into BY-2 cells with stable expression of GFP. Such observation was surprising because the IR-GFP was controlled by $X V E$ system that is considered to be one of the most reliable, i.e. the least suffering from leaky transcription $[25,26]$. Although it is rare in the XVE system, there are reports showing that leaky expression can occur $[39,40]$. To exclude this possibility, we employed additional controls; two IR-GFP constructs without the presence of the inducible (or any other) promoter. The results were identical in both cases (Fig. 2), clearly showing that the silencing did not occur as a result of the inducible promoter leakiness.

The observed silencing of the GFP clearly correlated with transcription of the IR-GFP, suggesting that silencing occurred as a result of this transcription at the posttranscriptional level (Fig. 3). By testing PIN3 inverted repeat, we excluded the possibility that the GFP 

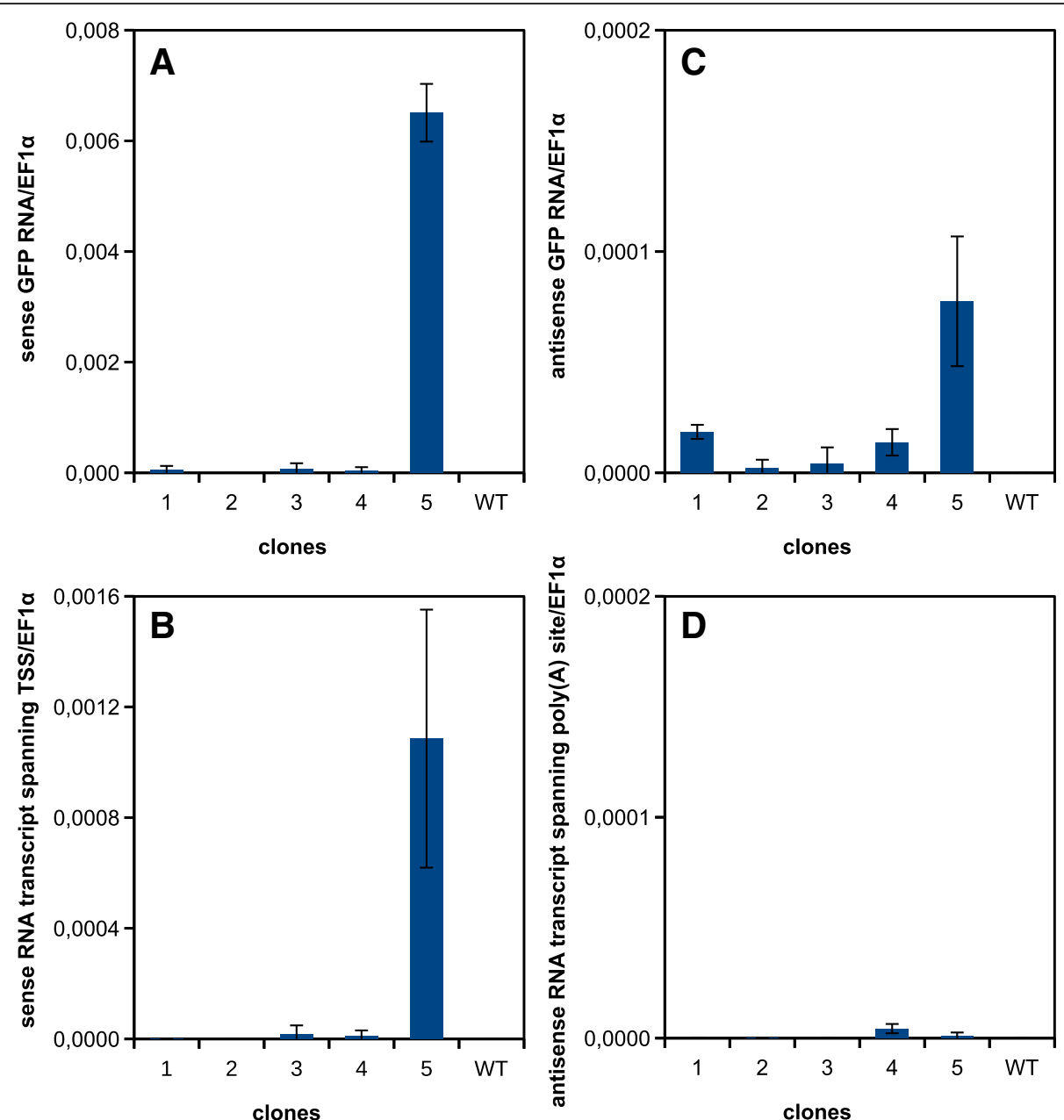

Fig. 7 Transcription of GFP gene in siliques of selected Arabidopsis thaliana transformants with pER8-GFP. RT-qPCR analysis of transcript levels in five selected plants untreated with $\beta$-estradiol. a The level of the sense GFP transcript (RT: R1 primer, QPCR: F1 and R1 primers); $\mathbf{b}$ the level of the sense transcript containing the region $50 \mathrm{nt}$ upstream of transcription start site (TSS) of the inducible promoter (RT: R1 primer, qPCR: F2 and R2 primers); $\mathbf{c}$ the level of the antisense GFP transcript (RT: F1 primer, QPCR: F1 and R1 primers); $\mathbf{d}$ the level of the antisense transcript containing the region 50 nt downstream of the last poly(A) signal of $T_{3 A}$ terminator (RT: F1 primer, QPCR: F3 and R3 primers)

sequence itself was the cause of the spontaneous transcription. The IR-PIN3 was also clearly transcribed in vast majority of analyzed calli, although we did not observe strong PIN3 silencing as in the case of GFP (Fig. 4). Repeat regions are often targets of silencing [13, 41], so we asked whether the repetitive nature of our silencing inducers (IR-GFP and IR-PIN3) could be the reason for why they were spontaneously transcribed. But RT-qPCR analysis showed that single sense GFP gene was transcribed at similar frequency and level in BY-2 calli, so we assumed that neither the repeat structure of the sequence was necessary for the spontaneous transcription (Fig. 5). Since equal frequency of GFP silencing was observed with two different T-DNAs carrying the $I R-G F P$, we could conclude that the spontaneous transcription was unlikely connected with any regulatory elements present within the T-DNAs.
Expression of endogenous PIN3 was more resistant against silencing induced by spontaneous transcription of IR-PIN3

Unlike IR-GFP, spontaneous transcription of IR-PIN3 resulted in only small decrease or unaffected level of PIN3 mRNA (Fig. 4 and Additional file 2). The level of IR-PIN3 transcription was somewhat lower than the transcription of IR-GFP, but the lowest transcript level of IR-GFP able to silence GFP expression was lower than the highest transcript level of IR-PIN3 that was not able to silence PIN3 expression (Figs. 3 and 4). The IR-PIN3 transcript could be theoretically less efficient in forming dsRNA and producing siRNAs. However, after treatment with $\beta$-estradiol, the same construct worked as an effective inducer of silencing (Jan Petrášek, personal communication). An alternative explanation is that the difference was related to the higher sensitivity of artificially introduced GFP transgene 
to silencing. This could be connected with the absence of introns in the GFP gene. The presence of an intron and its splicing can suppress production of secondary siRNAs that might be necessary to amplify silencing when the transcript level of the inducer is low [42-44].

\section{Pol II was involved in the spontaneous transcription}

Plant specific RNA Polymerases IV and V provide wide-spread transcription of genomic DNA important for de novo DNA methylation and maintenance of heterochromatin [45-47]. The transcripts we detected thus may originate from these polymerases. However, the size of the transcripts we detected exceeded the size typical for Pol IV and $\mathrm{V}$ transcripts, i.e. tens of $\mathrm{nt}$ long up to few hundreds nt respectively $[48,49]$. Moreover, at least some transcripts in the sense direction were spliced (Fig. 3g), which is typical for Pol II transcription. The ratio between the spliced and unspliced transcripts was similar in calli with spontaneous transcription and in estradiol-induced control line, where transcription was fully done by Pol II (compare columns e in Fig. 3c and g). The very low levels of spliced transcripts likely resulted from instant processing by the DCLs [13]. Amplification of transcripts from cDNA samples prepared by reverse transcription with oligo $\mathrm{dT}$ primers further indicates that at least a portion of the transcripts were polyadenylated (Additional file 5). Although we cannot exclude the possibility that other polymerases contributed to the spontaneous transcription, Pol II surely participated, because splicing and the presence of a polyA tail are characteristic features for its transcription.

\section{Pervasive character of spontaneous transcription was possibly connected with specific chromatin state of some T-DNAs}

The level of the spontaneous transcription strongly differed between independently transformed lines carrying the same T-DNA, indicating that the spontaneous transcription was not general, but rather specific to certain insertion events/loci.

Our attempts to find the transcription start site indicated that it was located outside of the T-DNA. It was previously demonstrated that transcripts originating from adjacent genomic regions could read-through across the border regions of T-DNA inserts and affect expression of transgenes present near the border sequence $[50,51]$. Such transcription can also originate from a neighboring T-DNA. For this to occur in our system, the T-DNAs would have to be arranged either as tandem direct repeats or as tail-to-tail inverted repeats. But, it seems unlikely, that two thirds of transformed lines would contain such T-DNA arrangement and also our Southern hybridization did not indicate increased presence of such arrangements among the silenced lines. Therefore, we hypothesize that it was just the higher
T-DNA copy number detected in silenced lines, which increased the probability that at least one T-DNA copy was inserted in a genomic region supporting the spontaneous transcription.

In the case of sense transcripts spanning the $p E R 8$ $T-D N A$, the polymerase was able to run across two terminators, yet the frequency of spontaneous transcription (silencing) was the same as in the case of the $p$ Green $T-D N A$, where IR-GFP was located near the border sequence. It should be also noted that the spontaneous transcriptional activity was often bidirectional, and that there was a correlation between the sense and antisense transcription levels in many calli. Based on these observations, we hypothesize that the spontaneous transcription was connected with specific chromatin state in some T-DNA insertion loci.

Our previous study indicated that the establishment of epigenetic marks can be accidental and at least in some cases independent of the chromosomal environment, since different epigenetic states could be established in the same insertion locus [24]. T-DNAs, when being inserted into the chromosome, are likely free of any epigenetic marks; as such, the formation of new marks may either reflect the chromatin state in the insertion locus or the new chromatin may be labelled with marks specific for new DNA insertions. This could make it prone to basal transcription by polymerase II that can somehow ignore terminators, thus forming long transcripts, irrespective of canonical transcription units present in the sequence. It is known, that facultative termination of long non-coding RNAs (lnRNAs) can be involved in regulation of gene expression and silencing [52-54] and that the chromatin state does have an effect on transcription termination [55]. More specifically, it was demonstrated that IBM2 allows Pol II to read-through silenced transposable elements inserted in introns of genes [56] and recent report showed that similar mechanism can also work for T-DNAs in introns of genes [57]. So we hypothesize that this long read-through transcription of newly integrated T-DNAs could be a component of genomic safety mechanisms evolved to allow silencing of newly integrated invasive DNAs (like transposable elements, TE). Such long polymerase II transcripts could be recognized by ubiquitous TEs-derived hc-siRNAs to initiate their silencing. Being an internal part of long transcript, there would be a little risk of translation of the TE transcript into executive proteins that would activate their replication/transposition.

\section{Read-through transcription of inserted T-DNAs might be a more general phenomenon}

The important question is how widespread and how significant this phenomenon can be. We tested three types of gene constructs (IR-GFP, IR-PIN3 and GFP) within 
two different T-DNAs ( $p E R 8$ and $p$ Green) using two different model organisms (BY-2 tobacco cell line and Arabidopsis thaliana plants). The results with various T-DNAs in BY-2 tobacco cell line were highly consistent, showing high frequency of read-through transcription and relatively high transcript levels. Additional analysis done with one T-DNA in Arabidopsis thaliana slightly differed, showing lower levels of transcription and lower occurrence among analyzed transformed plants. This could be connected with the physiological state of cells used for analyses (cell line vs. intact plant tissues) or different transcriptional regulation in species with dissimilar genome size (tobacco vs. Arabidopsis thaliana).

We also searched previous studies, which used inducible systems in plants to find further support for our observation. But most of the studies did not report the levels of RNA and if yes, then it is unclear if the transcript was not detected due to its absence or because it was below the detection limit. For example, Dohi et al. [23] did not detect any transcripts from the uninduced XVE system in BY-2 cell lines using Northern blot with DIG-labeled probes, but neither we were able to detect this transcription using the same detection method (data not shown), yet the RT-qPCR method clearly confirmed presence of these transcripts. In accord with our results, Kubo et al. [58] also detected low levels of transcripts from uninduced XVE system in Physcomitrella patens using RT-qPCR.

Additional strong support comes from early studies on silencing that used promoterless constructs as controls; they analyzed if silencing was caused by RNA or if it could be mediated directly by DNA-DNA interaction [11, 51, 59-62]; these works were done in Petunia, tobacco, Arabidopsis thaliana and Neurospora. Of the works mentioned above, only Cogoni et al. [51] partially characterized the origin of the transcripts causing the silencing in Neurospora crassa, by showing that they likely originated outside of the transgene. Whereas some studies did not observe silencing with promoterless constructs [63, 64], several other studies indeed reported induction of silencing by constructs or T-DNAs arranged as inverted repeats even without the presence of a promoter sequence $[11,59,60]$. All these old observations could be easily explained as read-through pervasive transcription of inverted repeats that produced hairpin RNAs.

Our data illustrate high incidence of pervasive read-through transcription of T-DNAs in BY-2 cells. These data are supported by very large numbers of analyzed independently transformed lines, 200 to 800 calli per each variant. In plants, only Sijen et al. [60] previously showed transcripts that likely originated from the silencer locus. As such, our report provides a considerable contribution to a more than fifteen years unsolved enigma, which has been experimentally overlooked with exception of some studies on DNAi [65]. We presume that the phenomenon of read-through transcription of T-DNAs is general, but specifically manifests only when working with $I R$, which has high potential to effectively induce silencing [14]. In contrast, low-level transcription of other constructs easily passes unnoticed, as the long-range character of transcripts disables translation into functional proteins that could visibly affect the phenotype.

\section{Conclusions}

We observed unexpectedly high frequency of read-through low-level transcription of several different T-DNAs in tobacco BY-2 cell lines and to some extent also in Arabidopsis thaliana. We show that this transcription was at least partially catalyzed by Pol II, which was able to read-through two standard terminators. We speculate that this unusual transcription was connected with establishment of specific chromatin state in some T-DNA insertions. Such read-through transcription could be for example a component of a safety mechanism for recognition and silencing of invasive DNA insertions. In the case of T-DNAs containing $I R-G F P$, the spontaneous read-through transcription was sufficient to initiate very efficient silencing of the GFP gene (in trans) in the majority of analyzed BY-2 calli. From the practical view, it is important that the researchers using inducible silencing systems should be aware of this phenomenon as it can in some cases largely affect the obtained results.

\section{Additional files}

\begin{abstract}
Additional file 1: List of primers used in this study. (XLS $13 \mathrm{~kb}$ )
Additional file 2: Comparison of mean transcript levels from the analyzed T-DNAs. (A) Means of the IR transcript levels presented in Fig. 3 and Fig. 4. The means were calculated separately for the spontaneously silenced independent calli (i.e. 5 biological replicates, marked -) and nonsilenced calli (marked + ), one additional non-silenced callus grown on the induction medium with $\beta$-estradiol (marked as " $\mathrm{e}^{\prime \prime}$ ) and on the control medium with DMSO (marked as " $\mathrm{d}^{\text {") }}$ is presented alongside. For the IR-PIN3, the category "spontaneously silenced calli" include half of the calli with the lowest expression of PIN3 mRNA, the rest of the calli is part of the "non-silenced calli" category. Note that the higher expression of IRPIN3 in the category "spontaneously silenced calli" is not statistically significant. (B) Means of the transcript levels presented in Fig. 5. In all the experiments, intron from the inverted repeat (for both IR-GFP and IR-PIN3) is amplified with the same set of primers, so direct comparison of the transcript levels is possible. Also all the GPCR data are corrected for PCR efficiency (see Methods), so approximate comparison of quantities for different transcripts is also possible. The error bars represent standard deviations. (PDF $63 \mathrm{~kb}$ )
\end{abstract}

Additional file 3: Southern hybridization of total genomic DNA from BY-2 calli transformed with pER8-IR-GFP. Silenced independent calli marked with "-" and non-silenced calli marked with "+" (see Fig. 3). A DIG-labelled probe of the HPT gene was hybridized with DNA cleaved by Nsil (N) and Asel (A). T-DNA copy number was estimated as the number of hybridizing bands. The presence of repeats was analyzed as follows: tandem direct repeat should give $6.8 \mathrm{kbp}$ fragment with both Nsil and Asel, plus one fragment of unknown size for Nsil and Asel; head-to-head inverted repeat should give $7.3 \mathrm{kbp}$ fragment when digested with Nsil and two fragments of unknown size when digested with Asel; tail-to-tail inverted repeat should give $9.6 \mathrm{kbp}$ fragment when digested with Asel 
and two fragments of unknown sizes when digested with Nsil. (PDF 34 $\mathrm{kb})$

Additional file 4: Transcription of GFP gene in leaves of selected Arabidopsis thaliana transformants with pER8-GFP. RT-qPCR analysis of transcript levels in five selected lines untreated with $\beta$-estradiol. (A) The level of the sense GFP transcript; (B) the level of the sense transcript containing the region 50 nt upstream of transcription start site (TSS) of the inducible promoter; (C) the level of the antisense GFP transcript; (D) the level of the antisense transcript containing the region $50 \mathrm{nt}$ downstream of the last poly(A) signal of $T_{3 A}$ terminator. (PDF $39 \mathrm{~kb}$ )

Additional file 5: Detection on polyA-tailed GFP transcripts in selected BY-2 calli transformed with $p$ ER8-GFP. Semiquantitative RT-PCR analysis of transcript levels in five independent calli untreated with $\beta$-estradiol (the same calli as in Fig. 5). cDNA was prepared using oligo dT primers. (A) The level of the Actin transcript (internal standard); (B) the level of the GFP transcript; (C) amplification of RNA samples that were not treated with reverse transcriptase to ensure that there was no DNA contamination. (PDF $41 \mathrm{~kb}$ )

\section{Abbreviations}

AS: antisense; IR: inverted repeat; LB: left border sequence of T-DNA; RB: right border sequence of T-DNA; UT: unterminated (without terminator)

\section{Acknowledgments}

We thank to Jan Petrášek for providing the IR-PIN3 construct, to Miloš Duchoslav for the intron from StPsbO gene and to Adéla Rủžičková for Arabidopsis plants transformed with an inducible GFP construct. We also thank to Kelly Hennessey for language corrections.

\section{Funding}

The work was supported by Ministry of Education, Youth and Sports of Czech Republic (project No. LO1417).

\section{Availability of data and materials}

The datasets generated during and/or analyzed during the current study are available from the corresponding author on reasonable request.

\section{Authors' contributions}

VC carried out all the experimental work. VC together with LF designed the study, interpreted the results and wrote the manuscript. Both authors read and approved the final manuscript.

\section{Ethics approval and consent to participate}

Not applicable.

\section{Consent for publication}

Not applicable.

\section{Competing interests}

The authors declare that they have no competing interests.

\section{Publisher's Note}

Springer Nature remains neutral with regard to jurisdictional claims in published maps and institutional affiliations.

\section{Received: 1 October 2018 Accepted: 12 October 2018} Published online: 22 October 2018

\section{References}

1. Gelvin SB. Integration of agrobacterium T-DNA into the plant genome. Annu Rev Genet. 2017;51:195-217.

2. Kim S-I, Veena, Gelvin SB. Genome-wide analysis of agrobacterium T-DNA integration sites in the Arabidopsis genome generated under non-selective conditions. Plant J Cell Mol Biol. 2007;51:779-91.

3. Shilo S, Tripathi P, Melamed-Bessudo C, Tzfadia O, Muth TR, Levy AA. TDNA-genome junctions form early after infection and are influenced by the chromatin state of the host genome. PLoS Genet. 2017;13:e1006875.
4. Alonso JM, Stepanova AN, Leisse TJ, Kim CJ, Chen H, Shinn P, et al. Genome-wide insertional mutagenesis of Arabidopsis thaliana. Science. 2003;301:653-7.

5. Koncz C, Martini N, Mayerhofer R, Koncz-Kalman Z, Körber H, Redei GP, et al. High-frequency T-DNA-mediated gene tagging in plants. Proc Natl Acad Sci. 1989:86:8467-71.

6. Hewelt A, Prinsen E, Schell J, Van Onckelen H, Schmülling T. Promoter tagging with a promoterless ipt gene leads to cytokinin-induced phenotypic variability in transgenic tobacco plants: implications of gene dosage effects. Plant J. 1994;6:879-91.

7. Hsing Y-I, Chern C-G, Fan M-J, Lu P-C, Chen K-T, Lo S-F, et al. A rice gene activation/knockout mutant resource for high throughput functional genomics. Plant Mol Biol. 2007;63:351-64.

8. Oltmanns H, Frame B, Lee L-Y, Johnson S, Li B, Wang K, et al. Generation of backbone-free, low transgene copy plants by launching T-DNA from the agrobacterium chromosome. Plant Physiol. 2010;152:1158-66.

9. Cluster PD, O'Dell M, Metzlaff M, Flavell RB. Details of T-DNA structural organization from a transgenic Petunia population exhibiting cosuppression. Plant Mol Biol. 1996;32:1197-203.

10. Kregten M van, Pater $S$ de, Romeijn R, Schendel R van, Hooykaas PJJ, Tijsterman M. T-DNA integration in plants results from polymerase- $\theta$ mediated DNA repair. Nat Plants 2016;2:16164.

11. Stam M, De Bruin R, Kenter S, Van Der Hoorn RAL, Van Blokland R, Mol JNM, et al. Post-transcriptional silencing of chalcone synthase in Petunia by inverted transgene repeats. Plant J. 1997;12:63-82.

12. Fojtová M, Bleys A, Bedrichová J, Houdt HV, Krrižová K, Depicker A, et al. The trans-silencing capacity of invertedly repeated transgenes depends on their epigenetic state in tobacco. Nucleic Acids Res. 2006;34:2280-93.

13. Bologna NG, Voinnet $O$. The diversity, biogenesis, and activities of endogenous silencing small RNAs in Arabidopsis. Annu Rev Plant Biol. 2014; 65:473-503.

14. Yan H, Chretien R, Ye J, Rommens CM. New construct approaches for efficient gene silencing in plants. Plant Physiol. 2006;141:1508-18.

15. Nagata T, Nemoto Y, Hasezawa S. Tobacco BY-2 cell line as the "HeLa" cell in the cell biology of higher plants. Int Rev Cytol. 1992;132:1-30.

16. Srba M, Černíková A, Opatrný Z, Fischer L. Practical guidelines for the characterization of tobacco BY-2 cell lines. Biol Plant. 2016;60:13-24.

17. Calarco JP, Borges F, Donoghue MTA, Van Ex F, Jullien PE, Lopes T, et al. Reprogramming of DNA methylation in pollen guides epigenetic inheritance via small RNA. Cell. 2012;151:194-205.

18. Ibarra CA, Feng X, Schoft VK, Hsieh T-F, Uzawa R, Rodrigues JA, et al. Active DNA demethylation in plant companion cells reinforces transposon methylation in gametes. Science. 2012;337:1360-4.

19. Lunerová-Bedřichová J, Bleys A, Fojtová M, Khaitová L, Depicker A, Kovařík A Trans-generation inheritance of methylation patterns in a tobacco transgene following a post-transcriptional silencing event. Plant J. 2008;54 1049-62.

20. Zhao M, San León D, Delgadillo MO, García JA, Simón-Mateo C. Virusinduced gene silencing in transgenic plants: transgene silencing and reactivation associate with two patterns of transgene body methylation. Plant J. 2014;79:440-52.

21. Butaye KMJ, Cammue BPA, Delauré SL, Bolle MFCD. Approaches to minimize variation of transgene expression in plants. Mol Breed. 2005; 16:79-91.

22. Dalakouras A, Tzanopoulou M, Tsagris M, Wassenegger M, Kalantidis K. Hairpin transcription does not necessarily lead to efficient triggering of the RNAi pathway. Transgenic Res. 2010;20:293-304.

23. Dohi K, Nishikiori M, Tamai A, Ishikawa M, Meshi T, Mori M. Inducible virusmediated expression of a foreign protein in suspension-cultured plant cells. Arch Virol. 2006;151:1075-84.

24. Nocarova E, Fischer L. Cloning of transgenic tobacco BY-2 cells; an efficient method to analyse and reduce high natural heterogeneity of transgene expression. BMC Plant Biol. 2009;9:44.

25. Moore I, Samalova M, Kurup S. Transactivated and chemically inducible gene expression in plants. Plant J. 2006;45:651-83.

26. Zuo J, Niu Q, Chua N. An estrogen receptor-based transactivator XVE mediates highly inducible gene expression in transgenic plants. Plant J. 2000;24:265-73.

27. Davis SJ, Vierstra RD. Soluble, highly fluorescent variants of green fluorescent protein (GFP) for use in higher plants. Plant Mol Biol. 1998;36: $521-8$. 
28. Hellens RP, Edwards EA, Leyland NR, Bean S, Mullineaux PM. pGreen: a versatile and flexible binary Ti vector for agrobacterium-mediated plant transformation. Plant Mol Biol. 2000;42:819-32.

29. Smith NA, Singh SP, Wang M-B, Stoutjesdijk PA, Green AG, Waterhouse PM Gene expression: Total silencing by intron-spliced hairpin RNAs. Nature. 2000; 407:319-20.

30. Murashige T, Skoog FA. Revised medium for rapid growth and bio assays with tobacco tissue cultures. Physiol Plant. 1962;15:473-97.

31. Deblaere R, Bytebier B, De Greve H, Deboeck F, Schell J, Van Montagu M, et al. Efficient octopine Ti plasmid-derived vectors for agrobacteriummediated gene transfer to plants. Nucleic Acids Res. 1985;13:4777-88.

32. Aranda PS, LaJoie DM, Jorcyk CL. Bleach gel: a simple agarose gel for analyzing RNA quality. Electrophoresis. 2012;33:366-9.

33. Bustin SA, Benes V, Garson JA, Hellemans J, Huggett J, Kubista M, et al. The MIQE guidelines: minimum information for publication of quantitative realtime PCR experiments. Clin Chem. 2009;55:611-22.

34. Ramakers C, Ruijter JM, Deprez RHL, Moorman AFM. Assumption-free analysis of quantitative real-time polymerase chain reaction (PCR) data. Neurosci Lett. 2003;339:62-6.

35. Dvořáková L, Srba M, Opatrny Z, Fischer L. Hybrid proline-rich proteins: novel players in plant cell elongation? Ann Bot. 2012;109:453-62.

36. Schmidt GW, Delaney SK. Stable internal reference genes for normalization of real-time RT-PCR in tobacco (Nicotiana tabacum) during development and abiotic stress. Mol Genet Genomics. 2010;283:233-41.

37. Nocarova E, Opatrny Z, Fischer L. Successive silencing of tandem reporter genes in potato (Solanum tuberosum) over 5 years of vegetative propagation. Ann Bot. 2010;106:565-72.

38. Ji G, Li L, Li QQ, Wu X, Fu J, Chen G, et al. PASPA: a web server for mRNA poly(A) site predictions in plants and algae. Bioinforma Oxf Engl. 2015;31:1671-3.

39. Degenhardt RF, Bonham-Smith PC. Arabidopsis ribosomal proteins RPL23aA and RPL23aB are differentially targeted to the nucleolus and are disparately required for Normal development. Plant Physiol. 2008;147:128-42.

40. Kang C-Y, Lian H-L, Wang F-F, Huang J-R, Yang H-Q. Cryptochromes, Phytochromes, and COP1 regulate light-controlled stomatal development in Arabidopsis. Plant Cell. 2009;21:2624-41.

41. Sasaki T, Lee T, Liao W-W, Naumann U, Liao J-L, Eun C, et al. Distinct and concurrent pathways of pol II and pol IV-dependent siRNA biogenesis at a repetitive trans-silencer locus in Arabidopsis thaliana. Plant J. 2014;79:127-38.

42. Christie M, Croft LJ, Carroll BJ. Intron splicing suppresses RNA silencing in Arabidopsis. Plant J. 2011;68:159-67.

43. Vaistij FE, Jones $L$, Baulcombe DC. Spreading of RNA targeting and DNA methylation in RNA silencing requires transcription of the target gene and a putative RNA-dependent RNA polymerase. Plant Cell. 2002;14:857-67.

44. Vermeersch L, De Winne N, Depicker A. Introns reduce transitivity proportionally to their length, suggesting that silencing spreads along the pre-mRNA. Plant J. 2010;64:392-401.

45. Johnson LM, Du J, Hale CJ, Bischof S, Feng S, Chodavarapu RK, et al. SRAand SET-domain-containing proteins link RNA polymerase $\mathrm{V}$ occupancy to DNA methylation. Nature. 2014;507:124-8.

46. Law JA, Du J, Hale CJ, Feng S, Krajewski K, Palanca AMS, et al. Polymerase IV occupancy at RNA-directed DNA methylation sites requires SHH1. Nature. 2013:498:385-9.

47. Wierzbicki AT, Cocklin R, Mayampurath A, Lister R, Rowley MJ, Gregory BD, et al. Spatial and functional relationships among pol V-associated loci, pol IV-dependent siRNAs, and cytosine methylation in the Arabidopsis epigenome. Genes Dev. 2012;26:1825-36.

48. Blevins T, Podicheti R, Mishra V, Marasco M, Wang J, Rusch D, et al. Identification of pol IV and RDR2-dependent precursors of 24 nt siRNAs guiding de novo DNA methylation in Arabidopsis. elife. 2015;4:e09591.

49. Wierzbicki AT, Haag JR, Pikaard CS. Noncoding transcription by RNA polymerase pol IVb/pol V mediates transcriptional silencing of overlapping and adjacent genes. Cell. 2008;135:635-48

50. Breyne P, Gheysen G, Jacobs A, Montagu MV, Depicker A. Effect of T-DNA configuration on transgene expression. Mol Gen Genet. 1992;235:389-96.

51. Cogoni C, Irelan JT, Schumacher M, Schmidhauser TJ, Selker EU, Macino G. Transgene silencing of the al-1 gene in vegetative cells of Neurospora is mediated by a cytoplasmic effector and does not depend on DNA-DNA interactions or DNA methylation. EMBO J. 1996;15:3153-63.

52. Mischo HE, Proudfoot NJ. Disengaging polymerase: terminating RNA polymerase II transcription in budding yeast. Biochim Biophys Acta BBA Gene Regul Mech. 2013;1829:174-85.
53. Touat-Todeschini L, Shichino Y, Dangin M, Thierry-Mieg N, Gilquin B, Hiriart E, et al. Selective termination of IncRNA transcription promotes heterochromatin silencing and cell differentiation. EMBO J. 2017;36:2626-41.

54. Liu F, Marquardt S, Lister C, Swiezewski S, Dean C. Targeted 3' processing of antisense transcripts triggers Arabidopsis FLC chromatin silencing. Science. 2010;327:94-7.

55. Morse NJ, Gopal MR, Wagner JM, Alper HS. Yeast terminator function can be modulated and designed on the basis of predictions of nucleosome occupancy. ACS Synth Biol. 2017;6:2086-95.

56. Saze H, Kitayama J, Takashima K, Miura S, Harukawa Y, Ito T, et al. Mechanism for full-length RNA processing of Arabidopsis genes containing intragenic heterochromatin. Nat Commun. 2013;4. https://doi.org/10.1038/ncomms3301.

57. Osabe K, Harukawa Y, Miura S, Saze H. Epigenetic regulation of Intronic transgenes in Arabidopsis. Sci Rep. 2017;7. https:/doi.org/10.1038/srep45166.

58. Kubo M, Imai A, Nishiyama $T$, Ishikawa M, Sato $Y$, Kurata $T$, et al. System for stable $\beta$-estradiol-inducible gene expression in the Moss Physcomitrella patens. PLoS One. 2013:8:e77356.

59. Luff $B$, Pawlowski $L$, Bender J. An inverted repeat triggers cytosine methylation of identical sequences in Arabidopsis. Mol Cell. 1999;3:505-11.

60. Sijen T, Vijn I, Rebocho A, van Blokland R, Roelofs D, Mol JNM, et al. Transcriptional and posttranscriptional gene silencing are mechanistically related. Curr Biol. 2001:11:436-40.

61. Van Blokland R, Van der Geest N, Mol JNM, Kooter JM. Transgene-mediated suppression of chalcone synthase expression in Petunia hybrida results from an increase in RNA turnover. Plant J. 1994;6:861-77.

62. Voinnet O, Vain P, Angell S, Baulcombe DC. Systemic spread of sequencespecific transgene RNA degradation in plants is initiated by localized introduction of ectopic Promoterless DNA. Cell. 1998;95:177-87.

63. Mette MF, Aufsatz W, van der Winden J, Matzke MA, Matzke AJM. Transcriptional silencing and promoter methylation triggered by doublestranded RNA. EMBO J. 2000;19:5194-201.

64. Waterhouse PM, Graham MW, Wang M-B. Virus resistance and gene silencing in plants can be induced by simultaneous expression of sense and antisense RNA. Proc Natl Acad Sci. 1998;95:13959-64.

65. Kawai-Toyooka H, Kuramoto C, Orui K, Motoyama K, Kikuchi K, Kanegae T, et al. DNA interference: a simple and efficient gene-silencing system for high-throughput functional analysis in the Fern Adiantum. Plant Cell Physiol. 2004;45:1648-57.
Ready to submit your research? Choose BMC and benefit from:
- fast, convenient online submission
- thorough peer review by experienced researchers in your field
- rapid publication on acceptance
- support for research data, including large and complex data types
- gold Open Access which fosters wider collaboration and increased citations
- maximum visibility for your research: over $100 \mathrm{M}$ website views per year
At BMC, research is always in progress. 